This item was submitted to Loughborough's Research Repository by the author.

Items in Figshare are protected by copyright, with all rights reserved, unless otherwise indicated.

\title{
Annihilation dynamics of molecular excitons measured at a single perturbative excitation energy
}

PLEASE CITE THE PUBLISHED VERSION

https://doi.org/10.1021/acs.jpclett.0c02141

\section{PUBLISHER}

American Chemical Society (ACS)

\section{VERSION}

AM (Accepted Manuscript)

\section{PUBLISHER STATEMENT}

This document is the Accepted Manuscript version of a Published Work that appeared in final form in Journal of Physical Chemistry Letters, copyright $@$ American Chemical Society after peer review and technical editing by the publisher. To access the final edited and published work see https://pubs.acs.org/doi/10.1021/acs.jpclett.0c02141.

\section{LICENCE}

CC BY-NC-ND 4.0

\section{REPOSITORY RECORD}

Heshmatpour, Constantin, Pavel Malevich, Felix Plasser, Maximilian Menger, Christoph Lambert, Frantisek Sanda, and Jürgen Hauer. 2020. "Annihilation Dynamics of Molecular Excitons Measured at a Single Perturbative Excitation Energy”. Loughborough University. https://hdl.handle.net/2134/12902357.v1. 


\title{
Annihilation Dynamics of Molecular Excitons Measured at a Single Perturbative Excitation Energy
}

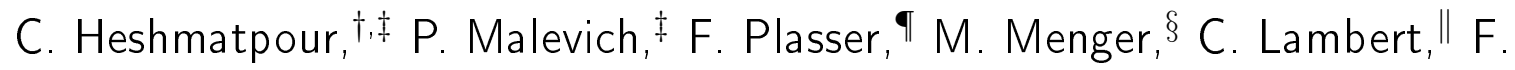 \\ Šanda, ${ }^{\dagger}$ and J. Hauer,
}

$\dagger$ Institute of Physics, Faculty of Mathematics and Physics, Charles University, Ke Karlovu 5, Prague 121 16, Czech Republic

$\ddagger$ Professur für Dynamische Spektroskopien, Fakultät für Chemie, Technische Universität

München, Lichtenbergstr. 4, D-85748, Garching b. München, Germany

ฯ Department of Chemistry, Loughborough University, Loughborough, LE11 3TU, United

Kingdom

$\S$ Faculty of Science and Engineering, Zernike Institute for Advanced Materials, University of Groningen, Nijenborgh 4, 9747AG Groningen, The Netherlands

|| Institut für Organische Chemie, Center for Nanosystems Chemistry, Universität

Würzburg, Am Hubland, 97074 Würzburg, Germany

E-mail: juergen.hauer@tum.de

\section{Abstract}

Exciton-exciton annihilation (EEA) is a ubiquitous phenomenon, which may limit the efficiency of photovoltaic devices. Conventional methods of determining EEA-timescales rely on measuring the intensity dependence of third order signals. In this work, we directly extract the annihilation rate of molecular excitons in a covalently joined molecular trimer without the need to perform and analyse intensity dependent data by employing fifth order coherent optical spectroscopy signals emitted into $\pm 2 \vec{k}_{1} \mp 2 \vec{k}_{2}+\vec{k}_{3}$ phase matching directions. Measured two-dimensional line shapes and their time traces are analysed in the framework of the many body dynamical version of the Frenkel exciton model, extended to incorporate annihilation dynamics. Combining double-sided Feynman diagrams with explicit simulations of the fifth order response, we identify a single peak as a direct reporter of EEA. We retrieve an annihilation time of 30 fs for the investigated squaraine trimer.

\section{Graphical TOC Entry}

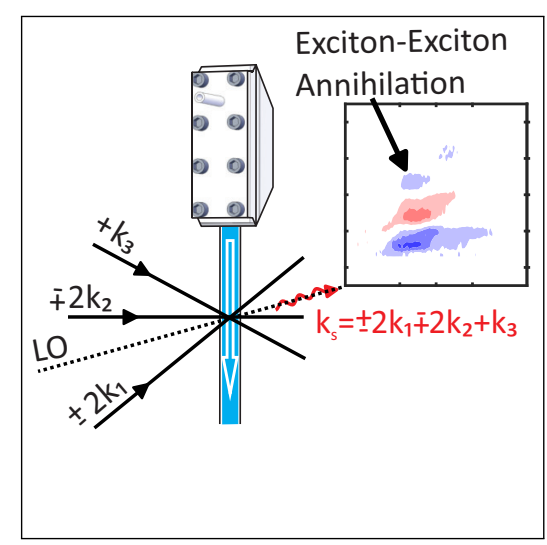

\section{Keywords}

Exciton-exciton annihilation, Multidimensional electronic spectroscopy, Fifth order two dimensional electronic spectroscopy, Exciton-excitoninteraction two-dimensional spectroscopy 


\section{Main Text}

Exciton-exciton annihilation (EEA) is the process of excitation energy quenching by fusion of two neighbouring excitons followed by nonradiative relaxation. ${ }^{1,2}$ EEA thus hampers the efficient conversion from light into usable energy in organic solar cells ${ }^{3,4}$ and is a limiting factor for lasers based on organic thin films ${ }^{5}$ by setting an upper limit for the exciton density. ${ }^{6}$ However, the efficient removal of excess energy via EEA is not solely undesirable as it, e.g plays an important role in the photoprotection of natural light harvesting complexes. ${ }^{7-10}$ EEA additionally explains the unconventional photocurrent generation efficiency of van der Waals heterostructure devices through the creation of hot holes. ${ }^{11}$ The most widespread application of EEA is to determine exciton diffusion constants as excitons need to migrate to adjacent sites to annihilate. ${ }^{12,13}$ Time-resolving annihilation dynamics thus allows on the one hand to identify undesirable loss channels and on the other hand it provides insights into the energy level structure of the material under study.

The investigation of annihilation dynamics is, however, experimentally challenging as it is commonly studied as an intensity dependent contribution to time-resolved third-order signals. ${ }^{14,15}$ Such approaches are prone to experimental artefacts at high excitation intensities, complicating the interpretation of the data. Fifth order 2D-electronic spectroscopy $\left(\mathrm{R}^{5}-2 \mathrm{D}\right)$ line shapes detected along $\vec{k}_{S}= \pm 2 \vec{k}_{1} \mp 2 \vec{k}_{2}+\vec{k}_{3}$ phase matching directions overcome this problem as bi-exciton dynamics, such as EEA, contribute to the signal already at perturbative level of excitation density. ${ }^{16-18}$ The general temporal evolution of the $\mathrm{R}^{5}-2 \mathrm{D}$ signal still contains both exciton transport and annihilation contributions. In a recent publication, ${ }^{19}$ we formally proved that spectral integration of fifth order signals over both, excitation and emission frequencies eliminates the dependence on single exciton transport rates for homo-aggregates with aligned transition dipole moment geometry of arbitrary length. The spectrally integrated $R^{5}-2 \mathrm{D}$ signal of such aggregates is thus a measure for tracking and pinpointing annihila- tion dynamics of molecular excitons at a single perturbative excitation density.

In this letter we extend our efforts towards aggregates with non-aligned transition dipoles and present a joint experimental and theoretical study of the $\mathrm{R}^{5}-2 \mathrm{D}$ signal of a squaraine homotrimer. To extract the annihilation dynamics of the trimer we investigate the observed line shapes in detail and identify a single peak as a direct reporter of EEA.

The experimental setup for measuring the $\mathrm{R}^{5}$ $2 \mathrm{D}\left(\vec{k}_{S}= \pm 2 \vec{k}_{1} \mp 2 \vec{k}_{2}+\vec{k}_{3}\right)$ signals is a fully noncollinear $50 \mathrm{kHz}$ shot-to-shot detected 2D electronic spectroscopy setup with sub 10 fs pulses in the visible. Our approach relies on conventional optics and represents an extension of our previously published setup ${ }^{20}$ for measuring $\mathrm{R}^{3}$ $2 \mathrm{D}\left(\vec{k}_{S}= \pm \vec{k}_{1} \mp \vec{k}_{2}+\vec{k}_{3}\right)$ signals. Fig. $1(\mathrm{~A})$ depicts the modified phase matching geometry enabling us to detect both $R^{3}$ - and $R^{5}-2 D$ signals within one experimental setup. A single 2D map was acquired in under four seconds employing the $50 \mathrm{kHz}$ shot-to-shot broadband detection and rapid scanning of coherence time $t_{1}$. A detailed explanation of the setup and the measurement procedure is presented in section 1 of the SI.

Measurements were performed on a squarainetrimer (tSQA, see Fig. 1(B)) in mesitylenesolution. This solvent was chosen for its high viscosity and low vapour pressure. Both parameters are important, as all time-resolved measurements were performed in a windowfree jet. ${ }^{21}$ tSQA was chosen as a paradigmatic strongly coupled trimeric system with high absorption cross section and spectra shown in Fig. 1C. ${ }^{22}$ Furthermore, a trimer is the smallest aggregate allowing for the full wealth of relaxation channels potentially affecting $\mathrm{R}^{5}-2 \mathrm{D}$ signals, including bi-exciton transport, while the system of electronic states required for the simulations of the non-linear signals is sufficiently small to hold a full many-body excitonic treatment. ${ }^{19}$

The experimental data of tSQA (absorption, $\mathrm{R}^{3}$ - and $\mathrm{R}^{5}-2 \mathrm{D}$ ) are thus compared against simulations employing a Frenkel exciton trimer model $^{23}$ developed in reference 19. Briefly, each squaraine molecule is considered as a two-level system with site energy $E$. Electronic coupling 
is considered between all chromophores. Strong coupling between the squaraine molecules requires to assume Redfield type of transport dynamics $^{24}$ between delocalized many-body eigenstates, such as single-exciton states $\left|a_{i}\right\rangle$ and bi-exciton states $\left|\alpha_{i}\right\rangle(i \in\{1,2,3\})$, obtained by diagonalization of the Frenkel exciton Hamiltonian. A schematic energy level diagram is shown in Fig. 2(A). EEA dynamics, which will be shown to be essential for the simulation of the $\mathrm{R}^{5}-2 \mathrm{D}$ data, are assumed to occur when neighbouring sites are occupied. EEA then leads to unidirectional transfer between manifolds, i.e. from tri-exciton state $|f\rangle \rightarrow|\alpha\rangle$ and $|\alpha\rangle \rightarrow|a\rangle$. The annihilation kinetics are Markovian and identical for both chromophore pairs. The linear and non-linear response of the trimer is calculated in the impulsive limit employing Kubo theory. ${ }^{25}$ We implemented a procedure to rotationally average absorption, $\mathrm{R}^{(3)}$ and $\mathrm{R}^{(5)}-2 \mathrm{D}$ signals along reference 26 . No additional line-broadening procedure has been applied, the calculated line shapes are thus purely homogeneous. Liouville space pathways, ${ }^{27}$ details and the parametrization of the model can be found in the SI.

The Frenkel exciton Hamiltonian (site energy and couplings) is parametrized by quantum chemical calculations with restrictions set by the tSQA absorption spectrum (see Fig. 1(C)). It displays a pronounced peak at $14100 \mathrm{~cm}^{-1}$, a weaker peak at $16100 \mathrm{~cm}^{-1}$ and a spectrally broad feature around $15000-16000 \mathrm{~cm}^{-1}$. The transition strength from the ground state into these single exciton states depends on the relative orientation of the transition dipoles associated with each chromophore and is thus linked to the trimer's conformation. ${ }^{28}$ From tSQA's molecular structure, shown in 1(B), one can expect the presence of several conformers, as rotations around the single bond connecting the monomeric units is conceivable. We thus performed exploratory quantum chemical calculations at the PBE0/SVP level of theory, ${ }^{29,30}$ suggesting seven relevant conformers at room temperature. These can be grouped into linear conformers, analogous to the structure shown in Fig. 1(B), as well as structures with one and two kinks. All conform-
(A)

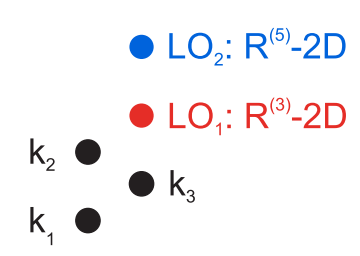

(B)
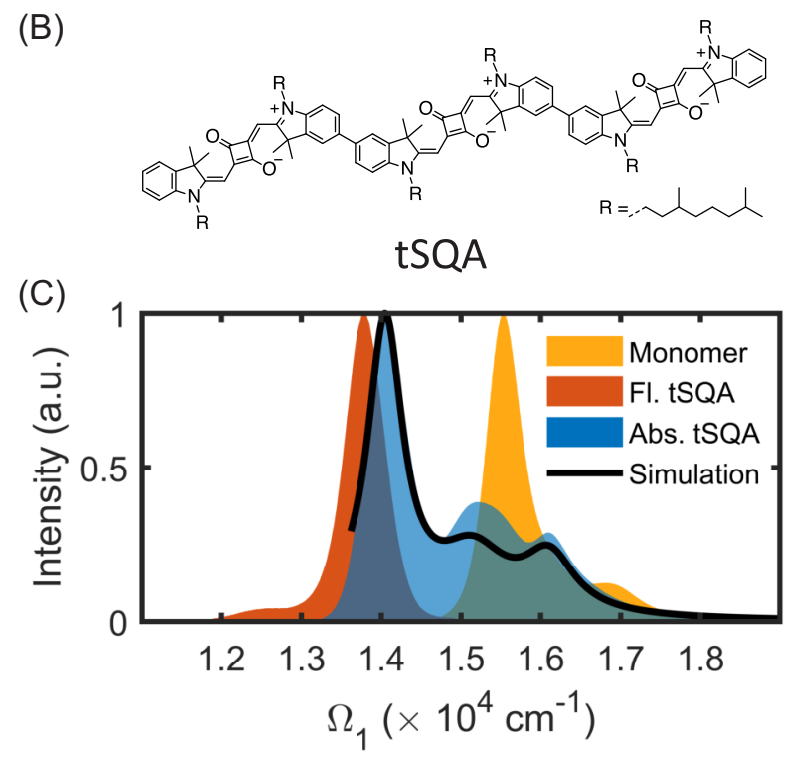

Figure 1: (A) Phase matching geometry used for heterodyne detection of $R^{(3)_{-}}$and $R^{(5)}{ }_{-} 2 \mathrm{D}$ signals of tSQA with local oscillators $L O_{1}$ and $L O_{2}$, respectively. Rephasing and non-rephasing signals were measured by interchanging the time-ordering of pulse $k_{1}$ and $k_{2}$. (B) tSQA's molecular structure. (C) Normalized linear spectra of tSQA and its monomer in mesitylene- and toluene-solution, respectively. The black solid line depicts the simulation of the absorption spectrum of tSQA employing the Frenkel exciton model described in the text.

ers are depicted in section 4 of the SI. We further computed the electronic couplings between the three chromophores of every structure by employing time-dependent density functional theory ${ }^{31,32}$ according to the scheme of Iozzi et al. ${ }^{33,34}$ implemented in Gaussian $16 .^{35}$ A comparison between single exciton level splittings of all conformers, observed in theory and experiment, revealed that the quantum chemistry based evaluations slightly underestimate the couplings. We thus scaled the couplings by $16 \%$, but kept their relative proportion. The scaled coupling data can be found in table 2 of the SI.

An effort to simultaneously fit all spectra, us- 
ing all suggested conformers with their associated coupling data and transition dipole geometries, revealed that the complete dataset can be adequately reproduced by an equal-weight linear combination employing the two conformers possessing one kink in their structure (structure number 5 and 6 in Fig. 4 of the SI). The resulting absorption spectrum is shown in $1(\mathrm{C})$ as a solid black line, which is in good agreement with the experiment. Differences between theory and experiment at the broad central feature are attributed to the in our calculation omitted minor vibronic feature visible in the monomer spectrum. The extent of vibronic contributions to the central broad peak of the trimer's absorption spectrum can be estimated by projecting the local $1300 \mathrm{~cm}^{-1}$ vibrations onto the lowest exciton $\left|a_{1}\right\rangle$. We estimate the amount of vibronic mixing by $d^{2} \sum_{i=1}^{3}\left|\left\langle i \mid a_{1}\right\rangle\right|^{4}, d^{2}=0.125$ is the monomer Huang Rhys factor. We find that the vibronic progression contributes less than $5 \%$ of the transition strength from the main absorption band for all structures, translating into a $10 \%$ vibronic contribution to the central peak volume. This is consistent with the observed deviation between experiment and theory as depicted in Fig. 1(C).

Fitting the absorption spectrum of tSQA adjusts, besides the Frenkel exciton Hamiltonian, the total peak broadenings as a sum of all pure dephasing and transport related broadenings. The $\mathrm{R}^{(3)}{ }_{-} 2 \mathrm{D}$ signal of tSQA is instrumental to distinguish between the two broadening mechanisms, by measuring dynamics inside the single exciton manifold. To keep the number of parameters tractable we derive the relaxation and decoherence rates from the environmental spectral density composed of an underdamped intramolecular mode at $1300 \mathrm{~cm}^{-1}$ identified in the monomer absorption spectrum (see Fig. $1(\mathrm{C})$ ) and a Lorentzian density for other vibrations and solvent modes. We thus used the $\mathrm{R}^{(3)}-2 \mathrm{D}$ signal to parametrize the spectral densities, or in other words to ensure that our parametrization captures the transport dynamics of tSQA. The result is depicted in section 5 of the SI.

As we now established a model for the transport dynamics of tSQA we can tackle the non- linear exciton dynamics which can be extracted from $\mathrm{R}^{(5)}-2 \mathrm{D}$ spectra. The respective signal of tSQA is depicted in Fig. 2(C) for two different waiting times. It shows an overall three peak structure, i.e. two negative and one positive peak. We discussed a similar peak structure in reference 19 for a strongly coupled model homotrimer with H-type coupling. The non-aligned case of tSQA is slightly different as additional transitions are optically allowed. However, we confirmed numerically that only one transition between manifolds $|g\rangle \rightarrow|a\rangle,|a\rangle \rightarrow|\alpha\rangle$ and $|\alpha\rangle \rightarrow|f\rangle$ is dominant in each case. The electronic coupling between chromophore 1 and 3 in tSQA is negligible, which means that tSQA can be described by the nearest neighbour Hamiltonian of reference 19 . We employ an excitonic coupling $J \approx 700 \mathrm{~cm}^{-1}$ as a good approximation for both relevant structures. The dominant transitions are from ground to the lowest single exciton eigenstate $\left|a_{1}\right\rangle$ (transition frequency $\omega_{g a_{1}} \approx E-\sqrt{2} J \approx 14110 \mathrm{~cm}^{-1}$ ), from $\left|a_{1}\right\rangle$ to the lowest bi-exciton state $\left|\alpha_{1}\right\rangle$ $\left(\omega_{a_{1} \alpha_{1}} \approx E \approx 15100 \mathrm{~cm}^{-1}\right)$ and from $\left|\alpha_{1}\right\rangle$ to tri-exciton state $|f\rangle\left(\omega_{\alpha_{1} f} \approx E+\sqrt{2} J \approx\right.$ $\left.16090 \mathrm{~cm}^{-1}\right)$. Three peaks are thus expected to appear at positions $\left(\Omega_{1}, \Omega_{3}\right)=\left(\omega_{g \alpha_{1}}, \omega_{g a_{1}}\right)$, $\left(\omega_{g \alpha_{1}}, \omega_{a_{1} \alpha_{1}}\right)$ and $\left(\omega_{g \alpha_{1}}, \omega_{\alpha_{1} f}\right)$, where $\omega_{g \alpha_{1}} \approx$ $2 E-\sqrt{2} J \approx 29210 \mathrm{~cm}^{-1}$ is the frequency of coherence $\left.\left|g \alpha_{1}\right\rangle\right\rangle$. The lowest negative feature thus stems predominantly from an interference between pathways in coherence $\left.\left|a_{1} g\right\rangle\right\rangle$ during $t_{3}$, the positive middle feature predominantly from pathways ending in $\left.\left|\alpha_{1} a_{1}\right\rangle\right\rangle$ and the negative feature at $15400 \mathrm{~cm}^{-1}$, termed 2Q-ESA in Fig. 2(C), predominantly from pathways in coherence $\left.\left|f \alpha_{1}\right\rangle\right\rangle$ (see Fig. 2(B)). The numbers obtained from the nearest neighbour model are in agreement with the simulation depicted in Fig. 2(D), which account for couplings between all units. The experimental peak positions in Fig. 2(C) are, however, slightly shifted down in $\Omega_{3}$ for early times compared to the nearest neighbour model and the according simulations shown in Fig. 2(D). Quantitative discrepancies in peak magnitudes between experiment and simulation (Fig. 2(D)) are attributed to overlaps of too-far reaching wings of simulated peaks, in other words to the Markovian approx- 
imation of the applied system kinetics. Despite these minor differences between Fig. 2(C) and (D) one sees that the electronic model describes the overall three peak structure and the varying intensities with increasing waiting time with good agreement. To extract the annihilation rate of tSQA from the signal we notice that the dominant diagrams for 2Q-ESA depicted in Fig. 2(B), require population of the bi-excitonic state during $t_{2}$. The strength of this negative contribution is thus a direct measure of population inside the bi-exciton manifold. Its disappearance directly relates to population relaxation out of the bi-exciton manifold, which determines the timescale for EEA. ${ }^{36}$ In Fig. 2 (E) we simulated the $\mathrm{R}^{(5)}-2 \mathrm{D}$ spectrum of $\mathrm{tSQA}$ without EEA to verify that the behaviour of the 2Q-ESA peak is a suitable reporter of EEA dynamics. The resulting 2D maps are distinctly changed, showing no major changes in the first few femtoseconds, despite the slight broadening along $\Omega_{1}$ of the lowest negative and the positive peak attributed to ultrafast excitation energy transfer. However, the 2Q-ESA peak shows no change, as population persists inside the bi-exciton manifold.

Fig. 3 depicts the experimental and simulated temporal evolution of the 2Q-ESA-peak's intensity in more detail. The experimental signal shows fast oscillations during pulse overlap ${ }^{37,38}$ and up to 20 fs followed by a rapid rise progressing into an oscillatory behaviour around its asymptotic value. The rapid rise of the signal is missing in simulations omitting annihilation and we thus assign it to EEA. The best agreement between theory and experiment in terms of $2 \mathrm{D}$ line shapes and temporal traces of the 2Q-ESA peak (see Fig. 3) is found with an annihilation time of $30 \mathrm{fs}$. The sensitivity of this multicriterial fit against $\tau_{A}$ is quantified by the mean of the residuals (MOR) of the fitted traces in Fig. 3 as compared to the experimental data; the choice of $\tau_{A}=30 \mathrm{fs}$ leads to a MOR of 0.01 with the residuals normally distributed around zero with no systematic deviations. Setting $\tau_{A}$ to 50 fs leads to a sixfold increase in MOR and a deviation of the residual from a zero average value (not shown). The 30 fs annihilation timescale is in agreement with the annihilation
(A) Energy levels

(B) Pathways
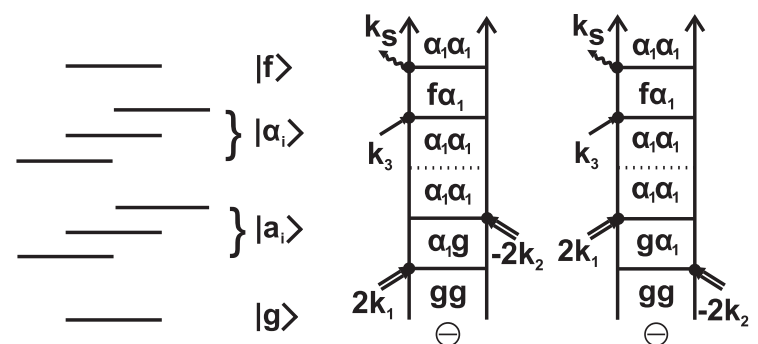

(C) Experiment

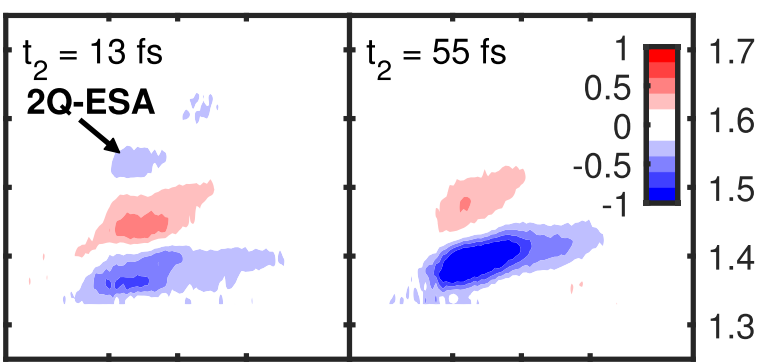

(D) Simulation: $\tau_{A}=30 \mathrm{fs}$

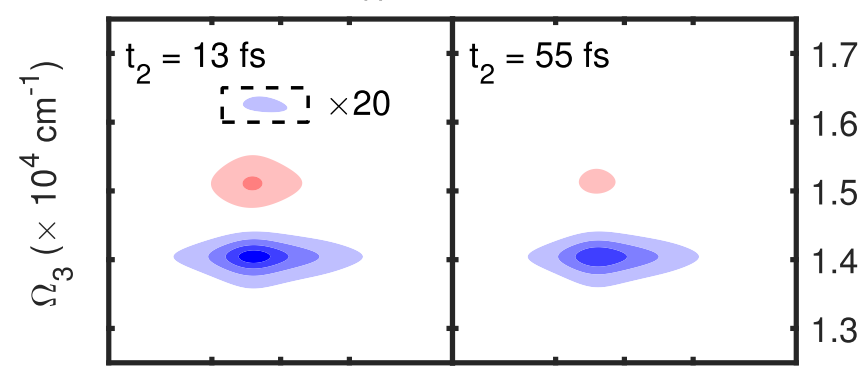

(E) Simulation: $\tau_{A} \rightarrow \infty$

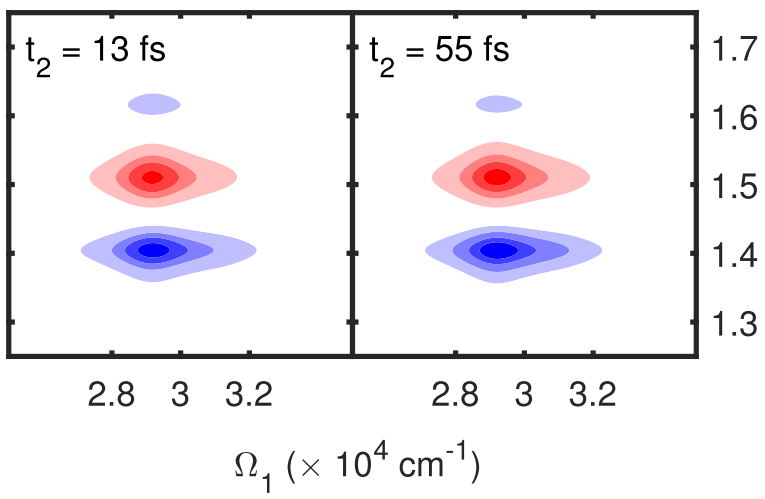

Figure 2: (A) tSQA energy level diagram. (B) Double-sided Feynman diagrams of the predominant contributions to peak 2Q-ESA. (C) Experimental $\mathrm{R}^{(5)}-2 \mathrm{D}$ spectra of tSQA for two different waiting times. (D,E) Simulations of the $R^{(5)}-2 \mathrm{D}$ signal for the same waiting times as in $(\mathrm{C})$, for an annihilation time $\tau_{A}$ of $30 \mathrm{fs}$ (D) and no annihilation between excitons (E). The peak in the dotted square is magnified by a factor of 20 for better visibility. The disappearance of the top negative peak, termed 2Q-ESA, for long waiting times is a direct reporter of EEA (see text). 


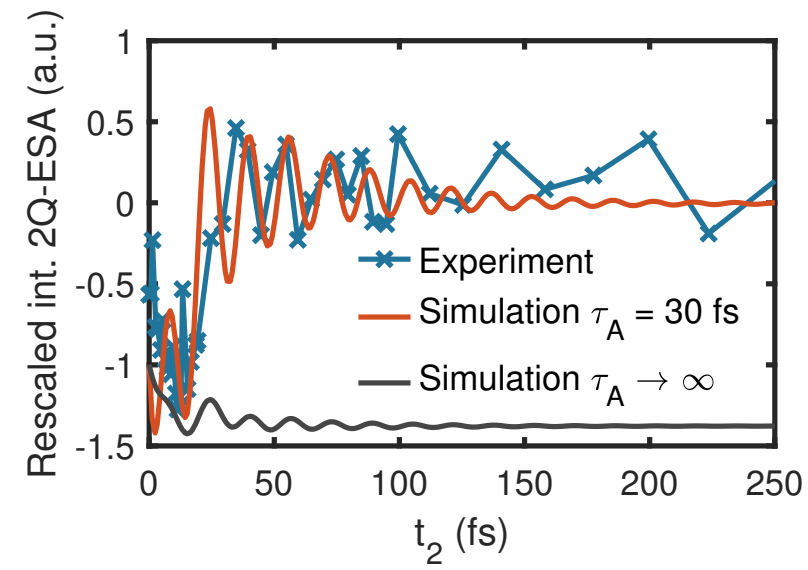

Figure 3: Experimental (blue) and simulated (red: $\tau_{A}=30$ fs, black: no EEA) intensity of peak 2QESA versus the waiting time $t_{2}$. The intensity is rescaled to start at -1 and to approach zero by subtracting an average value at long delays. Comparing all time traces we can associate the fast rise of the signal to EEA.

time of 28 fs found for a squaraine hetero-dimer (see SI of reference 18) following the temporal behaviour of a peak in fluorescence detected 2D-ES. ${ }^{39}$ The oscillations in the simulated signal $\left(\tau_{A}=30 \mathrm{fs}\right)$ in Fig. 3 are attributed to an excitonic beating in the first manifold, in particular between states $\left|a_{1}\right\rangle$ and $\left|a_{3}\right\rangle$ (see pathways R10, NR10, R8 and NR8 in Fig. 2 of SI). In this letter we presented a direct study of ultrafast annihilation dynamics of molecular excitons via $\mathrm{R}^{(5)}-2 \mathrm{D}$ spectroscopy. Spectroscopic measurements were performed on tSQA, a molecular trimer. The measured line shapes and time traces were explained by a developed theoretical model employing a Frenkel exciton Hamiltonian and Redfield theory for exciton dynamics, extended to incorporate EEA. Excitonic coupling and molecular structure were parametrized using quantum chemistry. The electronic model reproduces absorption, $\mathrm{R}^{(3)_{-}}$(see $\mathrm{SI}$ ) and $\mathrm{R}^{(5)}$ 2D spectra with good agreement. A possible, but computationally demanding extension of the electronic model towards a vibronic description, ${ }^{40,41}$ is left for future research. Employing double-sided Feynman diagrams, we identified a single peak as a reporter of EEA. Our analysis was consolidated by explicit simulations of the fifth order response of tSQA allowing and prohibiting EEA. The simulated time traces clearly show that the fast rise observed in the experiment is directly connected to EEA. Fitting the experimental data with our model we could extract an annihilation time of $30 \mathrm{fs}$. The obtained time constant is in accordance with measurements performed on aggregates of similar size, employing fluorescence detected 2DES (see SI of reference 18). In conclusion, we showed that $\mathrm{R}^{(5)}-2 \mathrm{D}$ presents a robust way of detecting bi-exciton dynamics, such as EEA, and thus circumvents the need to perform and analyse intensity dependent experiments.

Acknowledgement J.H, M.P and C.H. thank Harald Ceymann for providing and synthesizing the sample. J. H and C. H. acknowledge support by Deutsche Forschungsgemeinschaft (DFG, German Research Foundation) through TUM International Graduate School of Science and Engineering (IGSSE), GSC 81 and funding by DFG under Germany's Excellence Strategy - EXC 2089/1-390776260. F. Š and C.H. acknowledge support by Czech Science Foundation (Grant No. 17-22160S). Mobility is supported by project "Excitonexciton annihilation probed by non-linear spectroscopy" (MSMT Grant No. 8J19DE009, DAAD-Projekt 57444962). P.M. acknowledges the support of the Alexander von Humboldt Foundation as well as the donor, the Federal Ministry for Education and Research.

\section{Supporting Information Avail- able}

The following files are available free of charge.

- SI-R5-tSQA.pdf: Experimental setup, Model for optical dynamics of tSQA, Double-sided Feynman diagrams for $\mathrm{R}^{3}$ and $\mathrm{R}^{5}-2 \mathrm{D}$, tSQA structures, $\mathrm{R}^{3}-2 \mathrm{D}$ signal of tSQA

\section{References}

(1) Gaididei, Y. B.; Onipko, A. I. A Kinetic Theory of Incoherent Exciton Annihila- 
tion. Mol. Cryst. Liq. Cryst. 1979, 62, 213-235.

(2) May, V. Kinetic theory of exciton-exciton annihilation. J. Chem. Phys. 2014, 140, 054103 .

(3) Tzabari, L.; Zayats, V.; Tessler, N. Exciton annihilation as bimolecular loss in organic solar cells. J. Appl. Phys. 2013, 114, 154514 .

(4) McDonough, T. J.; Zhang, L.; Roy, S. S.; Kearns, N. M.; Arnold, M. S.; Zanni, M. T.; Andrew, T. L. Triplet exciton dissociation and electron extraction in graphene-templated pentacene observed with ultrafast spectroscopy. Phys. Chem. Chem. Phys. 2017, 19, 4809-4820.

(5) Baldo, M. A.; Holmes, R. J.; Forrest, S. R. Prospects for electrically pumped organic lasers. Phys. Rev. B 2002, 66, 035321.

(6) Yu, Y.; Yu, Y.; Xu, C.; Barrette, A.; Gundogdu, K.; Cao, L. Fundamental limits of exciton-exciton annihilation for light emission in transition metal dichalcogenide monolayers. Phys. Rev. B 2016, 93, 201111.

(7) Muller, M. G.; Lambrev, P.; Reus, M.; Wientjes, E.; Croce, R.; Holzwarth, A. R. Singlet energy dissipation in the photosystem II light-harvesting complex does not involve energy transfer to carotenoids. ChemPhysChem 2010, 11, 1289 - 1296.

(8) Valkunas, L.; van Stokkum, I. H. M.; Berera, R.; van Grondelle, R. Exciton migration and fluorescence quenching in LHCII aggregates: Target analysis using a simple nonlinear annihilation scheme. Chem. Phys. 2009, 357, $17-20$.

(9) Trinkunas, G.; Herek, J. L.; Polivka, T.; Sundstrom, V.; Pullerits, T. Exciton delocalization probed by excitation annihilation in the light-harvesting antenna LH2. Phys. Rev. Lett. 2001, 86, $4167-4170$.
(10) Valkunas, L.; Trinkunas, G.; Liuolia, V.; van Grondelle, R. Nonlinear annihilation of excitations in photosynthetic systems. Biophys. J. 1995, 69, $1117-1129$.

(11) Linardy, E.; Yadav, D.; Vella, D.; Verzhbitskiy, I. A.; Watanabe, K.; Taniguchi, T.; Pauly, F.; Trushin, M.; Eda, G. Harnessing Exciton-Exciton Annihilation in Two-Dimensional Semiconductors. Nano Lett. 2020, 20, 1647-1653.

(12) Fennel, F.; Lochbrunner, S. Excitonexciton annihilation in a disordered molecular system by direct and multistep Förster transfer. Phys. Rev. B 2015, 92.

(13) Nguyen, T.-Q.; Martini, I. B.; Liu, J.; Schwartz, B. J. Controlling Interchain Interactions in Conjugated Polymers: The Effects of Chain Morphology on ExcitonExciton Annihilation and Aggregation in MEH-PPV Films. J. Phys. Chem. B 2000, 104, 237-255.

(14) Bruggemann, B.; May, V. Exciton exciton annihilation dynamics in chromophore complexes. II. Intensity dependent transient absorption of the LH2 antenna system. J. Chem. Phys. 2004, 120, 2325-36.

(15) Valkunas, L.; Ma, Y.-Z.; Fleming, G. R. Exciton-exciton annihilation in singlewalled carbon nanotubes. Phys. Rev. B 2006, 73, 115432.

(16) Brüggemann, B.; Pullerits, T. Nonperturbative modeling of fifth-order coherent multidimensional spectroscopy in light harvesting antennas. New J. Phys. 2011, 13, 025024 .

(17) Dostál, J.; Fennel, F.; Koch, F.; Herbst, S.; Wurthner, F.; Brixner, T. Direct observation of exciton-exciton interactions. Nat. Commun. 2018, 9, 2466.

(18) Malý, P.; Lüttig, J.; Turkin, A.; Dostál, J.; Lambert, C.; Brixner, T. From wavelike to sub-diffusive motion: exciton dynamics and interaction in squaraine copolymers of 
varying length. Chem. Sci. 2020, 11, 456466.

(19) Heshmatpour, C.; Hauer, J.; Šanda, F. Interplay of exciton annihilation and transport in fifth order electronic spectroscopy. Chem. Phys. 2020, 528, 110433.

(20) Milota, F.; Lincoln, C. N.; Hauer, J. Precise phasing of 2D-electronic spectra in a fully non-collinear phase-matching geometry. Opt. Express 2013, 21, 15904-15911.

(21) Picchiotti, A.; Prokhorenko, V. I.; Miller, R. J. A closed-loop pump-driven wire-guided flow jet for ultrafast spectroscopy of liquid samples. Rev. Sci. Instrum. 2015, 86, 093105.

(22) Ceymann, H.; Rosspeintner, A.; Schreck, M. H.; Mutzel, C.; Stoy, A.; Vauthey, E.; Lambert, C. Cooperative enhancement versus additivity of two-photon-absorption cross sections in linear and branched squaraine superchromophores. Phys. Chem. Chem. Phys. 2016, 18, 16404-16413.

(23) Frenkel, J. On the Transformation of Light into Heat in Solids. II. Phys. Rev. 1931, 37, 1276-1294.

(24) Redfield, A. G. On the theory of relaxation processes. IBM J. Res. Dev. 1957, 1, $19-$ 31 .

(25) Kubo, R. Statistical-Mechanical Theory of Irreversible Processes. I. General Theory and Simple Applications to Magnetic and Conduction Problems. J. Phys. Soc. Jpn. 1957, 12, $570-586$.

(26) Andrews, D. L.; Thirunamachandran, T. On three-dimensional rotational averages. J. Chem. Phys. 1977, 67, 5026-5033.

(27) S.Mukamel, Principles of Nonlinear Optical Spectroscopy; OUP, 1995.

(28) Seibt, J.; Dehm, V.; Wurthner, F.; Engel, V. Absorption spectroscopy of molecular trimers. J. Chem. Phys. 2007, 126, 164308
(29) Adamo, C.; Barone, V. Toward reliable density functional methods without adjustable parameters: The PBE0 model. $J$. Chem. Phys. 1999, 110, 6158-6170.

(30) Weigend, F.; Ahlrichs, R. Balanced basis sets of split valence, triple zeta valence and quadruple zeta valence quality for $\mathrm{H}$ to Rn: Design and assessment of accuracy. Phys. Chem. Chem. Phys. 2005, 7, 32973305.

(31) Casida, M. E.; Jamorski, C.; Casida, K. C.; Salahub, D. R. Molecular excitation energies to high-lying bound states from time-dependent densityfunctional response theory: Characterization and correction of the time-dependent local density approximation ionization threshold. J. Chem. Phys. 1998, 108, 4439-4449.

(32) Yanai, T.; Tew, D. P.; Handy, N. C. A new hybrid exchange-correlation functional using the Coulomb-attenuating method (CAM-B3LYP). Chem. Phys. Lett. 2004, 393, 51-57.

(33) Iozzi, M. F.; Mennucci, B.; Tomasi, J.; Cammi, R. Excitation energy transfer (EET) between molecules in condensed matter: A novel application of the polarizable continuum model (PCM). J. Chem. Phys. 2004, 120, 7029-7040.

(34) Curutchet, C.; Mennucci, B. Quantum Chemical Studies of Light Harvesting. Chem. Rev. 2017, 117, 294-343.

(35) Frisch, M. J.; Trucks, G. W.; Schlegel, H. B.; Scuseria, G. E.; Robb, M. A.; Cheeseman, J. R.; Scalmani, G.; Barone, V.; Petersson, G. A.; Nakatsuji, H. et al. Gaussian 16 Revision B.01. 2016; Gaussian Inc. Wallingford CT.

(36) van Amerongen, H.; van Grondelle, R.; Valkunas, L. Photosynthetic Excitons; World Scientific, 2000. 
(37) Perlík, V.; Hauer, J.; Šanda, F. Finite pulse effects in single and double quantum spectroscopies. J. Opt. Soc. Am. B 2017, 34.

(38) Doa, T. N.; Chenb, L.; Belyaevc, A. K.; Tana, H.; Gelin, M. F. Pulse-shape effects in fifth-order multidimensional optical spectroscopy. Chem. Phys. 2018, 515, $119-128$.

(39) Malý, P.; Mančal, T. Signatures of Exciton Delocalization and Exciton-Exciton Annihilation in Fluorescence-Detected TwoDimensional Coherent Spectroscopy. $J$. Phys. Chem. Lett. 2018, 9, 5654-5659.

(40) Zhong, C.; Bialas, D.; Collison, C. J.; Spano, F. C. Davydov Splitting in Squaraine Dimers. J. Phys. Chem. C 2019, 123, 18734-18745.

(41) Perlík, V.; Šanda, F. Vibrational relaxation beyond the linear damping limit in two-dimensional optical spectra of molecular aggregates. J. Chem. Phys. 2017, $147,084104$. 